\title{
A Variable-Flavour-Number Scheme at NNLO
}

\author{
Robert S. Thorne \\ Cavendish Laboratory, University of Cambridge, Madingley Road, Cambridge, CB3 OHE, UK
}

\begin{abstract}
I present a formulation of a Variable Flavour Number Scheme for heavy quarks that is implemented up to NNLO in the strong coupling constant and may be used in NNLO global fits for parton distributions.
\end{abstract}

Keywords: QCD, Structure Functions, Heavy Quarks

PACS: $12.38 . \mathrm{Bx}, 13.60 . \mathrm{Hb}$

While up, down and strange quarks are treated as effectively massless partons, charm, bottom and top have to be regarded as heavy partons. There are two distinct regimes for these types of quarks. At low scales, $Q^{2} \sim m_{H}^{2}$, they are only created in the final state and described using the Fixed Flavour Number Scheme (FFNS)

$$
F_{i}\left(x, Q^{2}\right)=C_{i, k}^{F F}\left(Q^{2} / m_{H}^{2}\right) \otimes f_{k}^{n_{f}}\left(Q^{2}\right) .
$$

However, for $Q^{2} \gg m_{H}^{2}$, the coefficient functions contain large $\ln \left(Q^{2} / m_{H}^{2}\right)$ terms, spoiling the perturbative expansion. In this regime it is more appropriate to treat the quarks like massless partons, and the large $\ln \left(Q^{2} / m_{H}^{2}\right)$ terms are summed via the DGLAP evolution equations. The simplest recipe involving this regime is the Zero Mass Variable Flavour Number Scheme (ZMVFNS). This ignores all $\mathscr{O}\left(m_{H}^{2} / Q^{2}\right)$ corrections, i.e.

$$
F_{i}\left(x, Q^{2}\right)=C_{i, j}^{Z M V F} \otimes f_{j}^{n_{f}+1}\left(Q^{2}\right) .
$$

The partons in different flavour-number regions are related perturbatively,

$$
f_{k}^{n_{f}+1}\left(Q^{2}\right)=A_{j k}\left(Q^{2} / m_{H}^{2}\right) \otimes f_{k}^{n_{f}}\left(Q^{2}\right),
$$

where the perturbative matrix elements $A_{j k}\left(Q^{2} / m_{H}^{2}\right)$ containing $\ln \left(Q^{2} / m_{H}^{2}\right)$ terms guarantee the correct evolution for both descriptions. At LO, i.e. zeroth order in $\alpha_{S}$, the relationship between the two descriptions is trivial $-q(g)_{k}^{n_{f}+1}\left(Q^{2}\right) \equiv q(g)_{k}^{n_{f}}\left(Q^{2}\right)$. At NLO, i.e. first order in $\alpha_{S}\left(h^{+}\left(Q^{2}\right)=(h+\bar{h})\left(Q^{2}\right)\right)$,

$$
h^{+}\left(Q^{2}\right)=\frac{\alpha_{S}}{4 \pi} P_{q g}^{0} \otimes g^{n_{f}}\left(Q^{2}\right) \ln \left(\frac{Q^{2}}{m_{H}^{2}}\right), \quad g^{n_{f}+1}\left(Q^{2}\right)=\left(1-\frac{\alpha_{S}}{6 \pi} \ln \left(\frac{Q^{2}}{m_{H}^{2}}\right)\right) g^{n_{f}}\left(Q^{2}\right),
$$

i.e. the heavy flavour evolves from zero at $Q^{2}=m_{H}^{2}$ and the gluon loses corresponding momentum. It is natural to choose $Q^{2}=m_{H}^{2}$ as the transition point. At NNLO, i.e. second order in $\alpha_{S}$, there is much more complication

$$
f_{i}^{n_{f}+1}\left(Q^{2}\right)=\left(\frac{\alpha_{S}}{4 \pi}\right)^{2} \sum_{i j}\left(A_{i j}^{2,0}+A_{i j}^{2,1} \ln \left(Q^{2} / m_{H}^{2}\right)+A_{i j}^{2,2} \ln ^{2}\left(Q^{2} / m_{H}^{2}\right)\right) \otimes f_{j}^{n_{f}}\left(Q^{2}\right),
$$



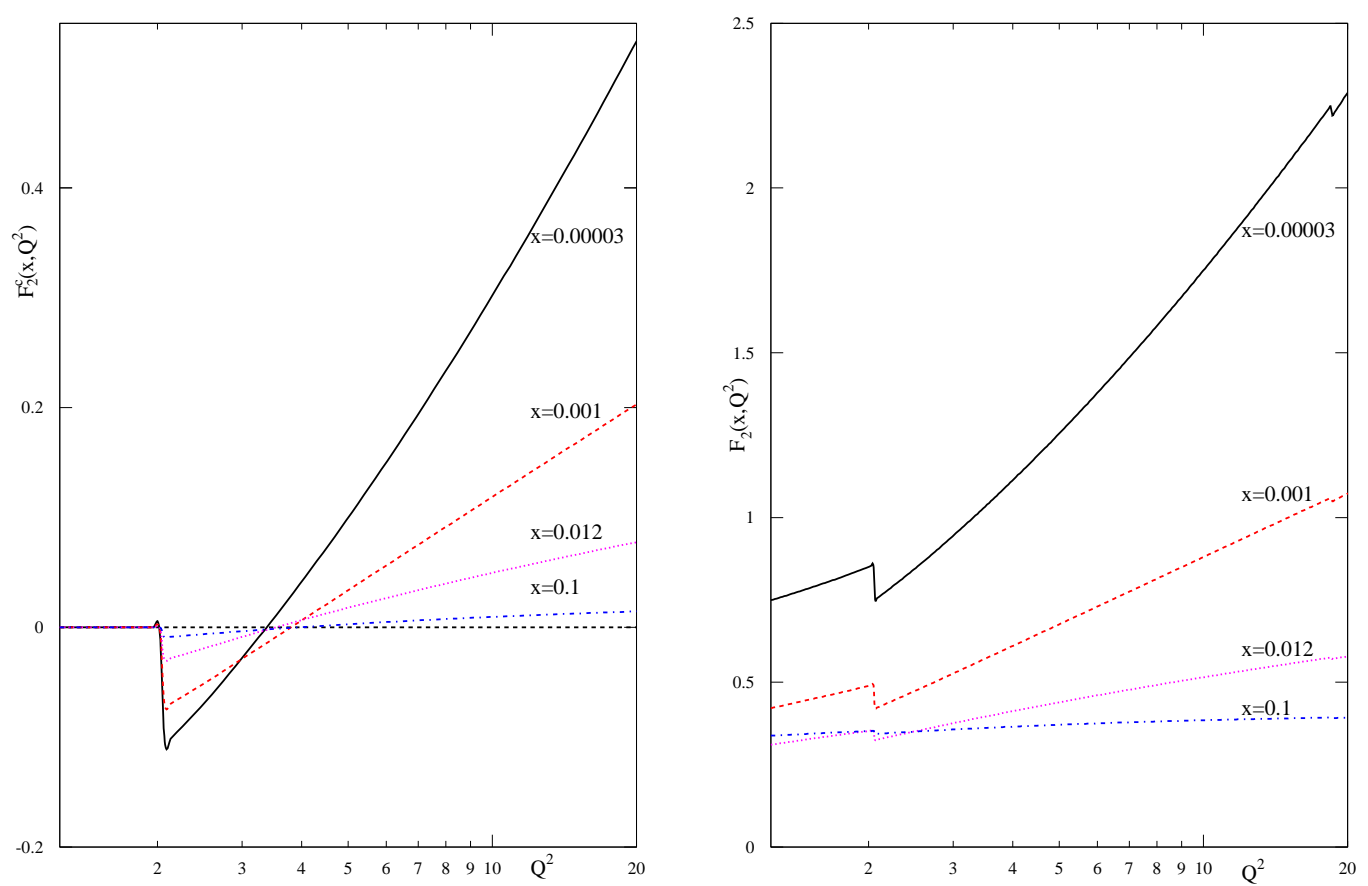

FIGURE 1. NNLO $F_{2}^{c}\left(x, Q^{2}\right)$ and $F_{2}\left(x, Q^{2}\right)$ in zero-mass VFNS

where $A_{i j}^{2,0}$ is generally non-zero [2]. There is no longer a smooth transition at this order, and in fact the heavy parton begins with a negative value at small $x$.

This leads to discontinuities in the partons and, without the correct treatment, also in the structure functions. ZMVFNS coefficient functions also lead to discontinuities at the transition point due to a sudden change in the flavour number in the coefficient functions. (This is already true at NLO, i.e. $F_{2}^{H}\left(x, Q^{2}\right)=0 \quad Q^{2}<m_{H}^{2},=\frac{\alpha_{S}}{4 \pi} C_{2, g} \otimes g^{n_{f}+1}\left(Q^{2}\right) Q^{2}>$ $m_{H}^{2}$, but the effect is very small.) This is a large effect at NNLO and is also negative at smallish $x$ ( $x \sim 0.001)$. Hence, ZMVFNS is not really feasible at NNLO, leading to a huge discontinuity in $F_{2}^{c}\left(x, Q^{2}\right)$, which is significant in $F_{2}^{T o t}\left(x, Q^{2}\right)$, as shown in Fig. 1.

Hence we need a general Variable Flavour Number Scheme (VFNS) interpolating between the two well-defined limits of $Q^{2} \leq m_{H}^{2}$ and $Q^{2} \gg m_{H}^{2}$. The VFNS can be defined by demanding equivalence of the $n_{f}$ and $n_{f}+1$-flavour descriptions at all orders,

$$
\begin{aligned}
F_{i}\left(x, Q^{2}\right) & =C_{i, k}^{F F}\left(Q^{2} / m_{H}^{2}\right) \otimes f_{k}^{n_{f}}\left(Q^{2}\right)=C_{i, j}^{V F}\left(Q^{2} / m_{H}^{2}\right) \otimes f_{j}^{n_{f}+1}\left(Q^{2}\right) \\
& \equiv C_{i, j}^{V F}\left(Q^{2} / m_{H}^{2}\right) \otimes A_{j k}\left(Q^{2} / m_{H}^{2}\right) \otimes f_{k}^{n_{f}}\left(Q^{2}\right) \\
\rightarrow C_{i, k}^{F F}\left(Q^{2} / m_{H}^{2}\right) & =C_{i, j}^{V F}\left(Q^{2} / m_{H}^{2}\right) \otimes A_{j k}\left(Q^{2} / m_{H}^{2}\right) .
\end{aligned}
$$

At $\mathscr{O}\left(\alpha_{S}\right)$ this gives

$$
C_{2, g}^{F F, 1}\left(Q^{2} / m_{H}^{2}\right)=C_{2, H H}^{V F, 0}\left(Q^{2} / m_{H}^{2}\right) \otimes P_{q g}^{0} \ln \left(Q^{2} / m_{H}^{2}\right)+C_{2, g}^{V F, 1}\left(Q^{2} / m_{H}^{2}\right) .
$$

The VFNS coefficient functions tend to the massless limits as $Q^{2} / m_{H}^{2} \rightarrow \infty$, as demonstrated to all orders in [3], and if we use the zeroth order cross-section for photon-heavy quark scattering we obtain the original ACOT scheme [1]. 
However, $C_{2, H H}^{V F, 0}\left(Q^{2} / m_{H}^{2}\right)$ is only uniquely defined as $Q^{2} / m_{H}^{2} \rightarrow \infty$, i.e. one can swap $\mathscr{O}\left(m_{H}^{2} / Q^{2}\right)$ terms between $C_{2, H H}^{V F, 0}\left(Q^{2} / m_{H}^{2}\right)$ and $C_{2, g}^{V F, 1}\left(Q^{2} / m_{H}^{2}\right)$. Similar reasoning holds for $C_{2, H H}^{V F, n}\left(Q^{2} / m_{H}^{2}\right)$. The ACOT prescription violated the threshold $W^{2}=Q^{2}(1-x) / x>$ $4 M^{2}$ since only one quark was needed in final state. The Thorne-Roberts variable flavour number scheme (TR-VFNS) [4] recognized this ambiguity and removed it by imposing continuity of $\left(d F_{2} / d \ln Q^{2}\right)$ at the transition point. This guaranteed smoothness at $Q^{2}=$ $m_{H}^{2}$, but was complicated and cumbersome when extended to higher orders.

There have been other alternatives, and most recently the ACOT $(\chi)$ prescription [5] defines $F_{2}^{H, 0}\left(x, Q^{2}\right)=h^{+}\left(x / x_{\max }, Q^{2}\right)$, where $x_{\max }=Q^{2} /\left(Q^{2}+4 m_{H}^{2}\right)$. The coefficient functions tend to the massless limit for $Q^{2} / m_{H}^{2} \rightarrow \infty$ but also respect the threshold requirement $W^{2} \geq 4 m_{H}^{2}$ for quark-antiquark production. Moreover it is very simple. For the VFNS to remain simple (and physical) at all orders I choose $C_{2, H H}^{V F, n}\left(Q^{2} / m_{H}^{2}, z\right)=$ $C_{2, H H}^{Z M, n}\left(z / x_{\max }\right) \cdot{ }^{1}$ Adopting this convention then at NNLO we have, for example,

$C_{2, H g}^{V F, 2}\left(\frac{Q^{2}}{m_{H}^{2}}\right)=C_{2, H g}^{F F, 2}\left(\frac{Q^{2}}{m_{H}^{2}}\right)-C_{2, H H}^{Z M, 1}\left(\frac{z}{x_{\max }}\right) \otimes A_{H g}^{1}\left(\frac{Q^{2}}{m_{H}^{2}}\right)-C_{2, H H}^{Z M, 0}\left(\frac{z}{x_{\max }}\right) \otimes A_{H g}^{2}\left(\frac{Q^{2}}{m_{H}^{2}}\right)$.

Since $A_{H g}^{2}(1, z) \neq 0, C_{2, H g}^{2}\left(Q^{2} / m_{H}^{2}, z\right)$ is discontinuous at $Q^{2}=m_{H}^{2}$, and this compensates exactly for the discontinuity in the heavy flavour parton distribution. ${ }^{2}$

There is one more issue in defining the VFNS: the ordering for $F_{2}^{H}\left(x, Q^{2}\right)$, i.e.

LO

$$
n_{f} \text {-flavour }
$$$$
n_{f}+1 \text {-flavour }
$$

$$
\frac{\alpha_{S}}{4 \pi} C_{2, H g}^{F F, 1} \otimes g^{n_{f}}
$$$$
C_{2, H H}^{V F, 0} \otimes h^{+}
$$

NLO

$$
\left(\frac{\alpha_{S}}{4 \pi}\right)^{2}\left(C_{2, H g}^{F F, 2} \otimes g^{n_{f}}+C_{2, H q}^{F F, 2} \otimes \Sigma^{n_{f}}\right)
$$$$
\frac{\alpha_{S}}{4 \pi}\left(C_{2, H H}^{V F, 1} \otimes h^{+}+C_{2, H g}^{F F, 1} \otimes g^{n_{f+1}}\right) .
$$

Switching directly when going from $n_{f}$ to $n_{f}+1$ flavours leads to a discontinuity. We must decide how to deal with this. Up to now ACOT have used e.g. at NLO

$$
\frac{\alpha_{S}}{4 \pi} C_{2, H g}^{F F, 1} \otimes g^{n_{f}} \rightarrow \frac{\alpha_{S}}{4 \pi}\left(C_{2, H H}^{V F, 1} \otimes h^{+}+C_{2, H g}^{F F, 1} \otimes g^{n_{f}+1}\right)+C_{2, H H}^{V F, 0} \otimes h^{+},
$$

i.e. the same order of $\alpha_{S}$ above and below, but LO below and NLO above. The ThorneRoberts scheme proposed e.g. at LO

$$
\frac{\alpha_{S}\left(Q^{2}\right)}{4 \pi} C_{2, H g}^{F F, 1}\left(\frac{Q^{2}}{m_{H}^{2}}\right) \otimes g^{n_{f}}\left(Q^{2}\right) \rightarrow \frac{\alpha_{S}\left(m_{H}^{2}\right)}{4 \pi} C_{2, H g}^{F F, 1}(1) \otimes g^{n_{f}}\left(m_{H}^{2}\right)+C_{2, H H}^{V F, 0}\left(\frac{Q^{2}}{m_{H}^{2}}\right) \otimes h^{+}\left(Q^{2}\right)
$$

i.e. the higher order $\alpha_{S}$ term is frozen when going upwards through $Q^{2}=m_{H}^{2}$. This difference in choice is extremely important at low $Q^{2}$.

${ }^{1}$ It is also important to choose $C_{L, H H}^{V F, n}\left(Q^{2} / m_{H}^{2}, z\right) \propto C_{L, H H}^{Z M, n}\left(z / x_{\max }\right)$.

2 At NNLO there are also contributions due to heavy flavours in loops away from the photon vertex. These are included within the VFNS and lead to a discontinuity in the coefficient functions for light flavours cancelling that in the light quark distributions. Strictly, part of this contribution should be interpreted as light flavour structure functions, while part of it contributes to $F_{2}^{H}\left(x, Q^{2}\right)[8]$. 

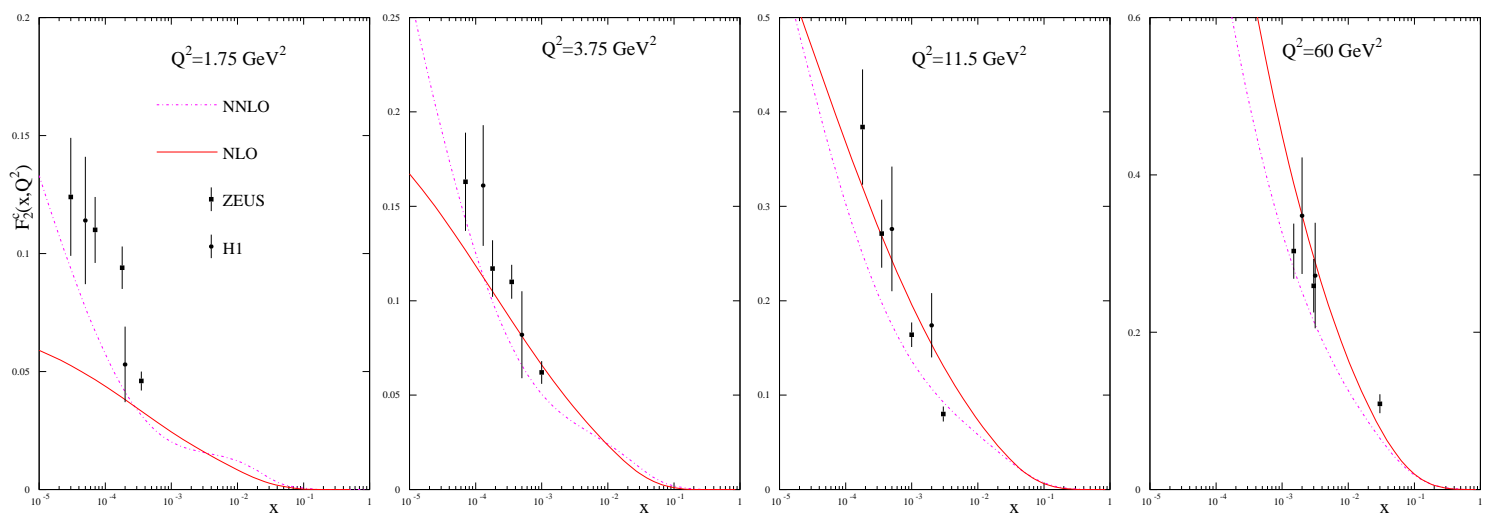

FIGURE 2. Comparison of NLO and NNLO predictions for $F_{2}^{c}\left(x, Q^{2}\right)$

Making this choice, in order to define the VFNS at NNLO we need the $\mathscr{O}\left(\alpha_{S}^{3}\right)$ heavy flavour coefficient functions for $Q^{2} \leq m_{H}^{2}$. However, these are not yet calculated (making a NNLO FFNS problematic). We know the leading threshold logarithms [6], and can derive the leading $\ln (1 / x)$ term from $k_{T}$-dependent impact factors [7],

$$
C_{2, H g}^{F F, 3, \text { lowx }}\left(Q^{2} / m_{H}^{2}, z\right)=96 \frac{\ln (1 / z)}{z} f\left(Q^{2} / m_{H}^{2}\right), \quad f(1) \approx 4,
$$

and $C_{2, H q}^{F F, 3, \text { lowx }}\left(Q^{2} / m_{H}^{2}, z\right)=4 / 9 C_{2, H g}^{F F, 3, \text { lowx }}\left(Q^{2} / m_{H}^{2}, z\right)$. By analogy with the known NNLO coefficient functions and splitting functions I hypothesize that

$$
C_{2, H g}^{F F, \text { lowx }}\left(Q^{2} / m_{H}^{2}, z\right)=\frac{96}{z}(\ln (1 / z)-4)\left(1-z / x_{\max }\right)^{20} f\left(Q^{2} / m_{H}^{2}\right),
$$

i.e. the leading $\ln (1 / z)$ term is always accompanied by $\sim-4$, and the effect of the small $z$ term is damped as $z \rightarrow 1$. Using the full (if slightly approximate) VFNS one can produce NNLO predictions for charm with discontinuous partons, but a continuous $F^{c}\left(x, Q^{2}\right)$. NNLO clearly improves the match to lowest $Q^{2}$ data [9, 10], where NLO is generally too low, as seen in Fig. 2.

Hence, I have devised a full NNLO VFNS, with a small amount of necessary modelling. This seems to improve the fit to the lowest $x$ and $Q^{2}$ data greatly. It also guarantees continuity of the physical observables, such as structure functions, despite the discontinuity in NNLO parton distributions. It can now be used in a full NNLO global analysis.

\section{REFERENCES}

1. M. Aivazis, F. Olness and W. K. Tung, Phys. Rev. D50 3102 (1994).

2. M. Buza, et al., Eur. Phys. J. C1 301 (1998).

3. J. C. Collins, Phys. Rev. D58 2000 (1998).

4. R. S. Thorne and R. G. Roberts, Phys. Lett. B421 303 (1998); Phys. Rev. D57 6871 (1998).

5. W. K. Tung et al., J. Phys. G28 983 (2002); S. Kretzer et al., Phys. Rev. D69 114005 (2004).

6. E. Laenen and S. Moch, Phys. Rev. D59 034027 (1999).

7. S. Catani, M. Ciafaloni and F. Hautmann, Nucl. Phys. B366 135 (1991).

8. A. Chuvakin, J. Smith and W. L. van Neerven, Phys. Rev. D61 096004 (2000).

9. ZEUS collaboration: S. Chekanov et al., Phys. Rev. D69 012004 (2004).

10. H1 collaboration: C. Adloff et al., Phys. Lett. B528 199 (2002). 